\title{
Bei Angst und Ärger steigt das Zytokin IL-6 im Blut an
}

\section{Bei akutem Psychostress lassen sich im Blut der Betroffenen Entzün- \\ dungsreaktionen nachweisen. Diese Reaktionen könnten an stressasso- ziierten Krankheiten beteiligt sein.}

- Forscher der University of Pittsburgh (USA) haben bei 102 Gesunden (mittleres Alter 50 Jahre, 60\% Frauen, 91,25\% Weiße) ein akutes Stressprotokoll durchgeführt. Der Stress wurde ausgelöst, indem man die Probanden vor einer Jury bei laufender Kamera eine Rede halten ließ. Nach einer einleitenden 30-minütigen Ruhephase, einer fünfminütigen Rede und einer erneuten, abschließenden 30-minütigen Ruhephase wurden jeweils Blutspiegel des proinflamma- torischen Zytokins IL- 6 bestimmt. Nach der Rede wurden die Probanden gefragt, wie sie sich fühlten.

Das IL-6 verhielt sich individuell sehr unterschiedlich. Die Konzentration war nicht assoziiert mit Veränderungen der Herzfrequenz und des Blutdrucks. Probanden, die während ihrer Rede Angst und/oder Ärger verspürten, reagierten aber mit einem signifikanten Anstieg des Interleukins.

\section{Kommentar}

Die Resultate zeigen, dass Menschen, die sich schnell ärgern oder ängstigen, mit einem Anstieg eines Entzündungsparameters reagieren. Häufiger Stress könnte bei ihnen also zu entzündlichen Erkrankungen
Angst und Ärger hinterlassen Spuren im Blut.

führen, etwa Herz-Kreislauf-Erkrankungen. Die Forscher wollen jetzt herausfinden, warum bestimmte Menschen auf Stress mit einer Mehrbildung von IL-6 reagieren und andere nicht.

K. MALBERG =

\section{- J. E. Carroll et al.}

(Behavioural Immunology Laboratory, Department of Psychology, University of Pittsburgh, USA; Negative affective responses to a speech task predict changes in interleukin (IL)-6.

Published in Access Online 25, Issue 2, February 2011: 232-238 